\title{
Do Outcomes in Elective Colon and Rectal Cancer Surgery Differ by Weekday? An Observational Study Using Data From the Dutch ColoRectal Audit
}

Daniëlle D. Huijts, MSca; Onno R. Guicherit, MD, PhD ; Jan Willem T. Dekker, MD, PhDc; Julia T. van Groningen, MD'; Leti van Bodegom-Vos, PhDa; Esther Bastiaannet, PhDi; Johannes A. Govaert, MD, PhDf;

Michel W. Wouters, MD, PhD ${ }^{\mathrm{d}, \mathrm{g}}$; and Perla J. Marang-van de Mheen, $\mathrm{PhD}^{\mathrm{a}}$

\section{ABSTRACT}

Background: Previous studies showing higher mortality after elective surgery performed on a Friday were based on administrative data, known for insufficient case-mix adjustment. The goal of this study was to investigate the risk of adverse events for patients with colon and rectal cancer by day of elective surgery using clinical data from the Dutch ColoRectal Audit. Patients and Methods: Prospectively collected data from the 2012-2015 Dutch ColoRectal Audit $(n=36,616)$ were used to examine differences in mortality, severe complications, and failure to rescue by day of elective surgery (Monday through Friday). Monday was used as a reference, analyses were stratified for colon and rectal cancer, and case-mix adjustments were made for previously identified variables. Results: For both colon and rectal cancer, crude mortality, severe complications, and failure-to-rescue rates varied by day of elective surgery. After case-mix adjustment, lower severe complication risk was found for rectal cancer surgery performed on a Friday (odds ratio, $0.84 ; 95 \% \mathrm{Cl}, 0.72-0.97$ ) versus Monday. No significant differences were found for colon cancer surgery performed on different weekdays. Conclusions: No weekday effect was found for elective colon and rectal cancer surgery in the Netherlands. Lower severe complication risk for elective rectal cancer surgery performed on a Friday may be caused by patient selection.

J Natl Compr Canc Netw 2019;17(7):821-828 doi: $10.6004 /$ jnccn.2018.7282

${ }^{a}$ Department of Biomedical Data Sciences, Medical Decision Making, Leiden University Medical Center, Leiden; ${ }^{b}$ Department of Surgery, University Cancer Center Leiden I The Hague, The Hague; 'Department of Surgery, Reinier de Graaf Group, Delft; ${ }^{d}$ Dutch Institute for Clinical Auditing, Leiden; ${ }^{\mathrm{e}}$ Department of Surgery, Leiden University Medical Center, Leiden; 'Department of Surgery, Groene Hart Ziekenhuis, Gouda; and 9Department of Surgical Oncology, Netherlands Cancer Institute-Antoni van Leeuwenhoek, Amsterdam, the Netherlands.

\section{Background}

Although surgery has a crucial role in colorectal cancer (CRC) treatment, it is associated with considerable risks of postoperative complications and mortality ${ }^{1,2}$ and a resultant increase in healthcare costs. ${ }^{3}$ Severe complications (ie, leading to prolonged hospital stay, reintervention, or death) have been reported in $18.3 \%$ of patients after elective colon cancer surgery and $24.8 \%$ after elective rectal cancer surgery. ${ }^{2}$ However, failure to rescue (FTR; death occurring after a severe complication) was higher for elective colon cancer surgery than for rectal cancer surgery ( $18.6 \%$ vs $9.4 \%$, respectively). ${ }^{2}$ Therefore, reducing the risk of adverse events might be of great importance for patients and healthcare institutions.

There are indications that these adverse events might be different depending on the days on which the elective surgery is performed. ${ }^{4}$ A recent study analyzing elective high-risk surgeries, including colorectal surgery, clearly observed a trend across weekdays, with significantly higher mortality rates for surgeries performed toward the end of the week. ${ }^{5}$ Adjusted odds ratios of 1.49 and 2.99, respectively, were observed for mortality when elective colorectal surgery occurred on a Friday or during weekends compared with Monday. The former results, the so-called weekend effect, should be interpreted with caution in the case of elective surgery. In the Netherlands, for example, elective surgery during weekends is not common practice, which could mean that only a specific group of patients is scheduled for surgery during the weekends (ie, selection bias) or that there was a coding error. Weekend elective surgeries are therefore excluded from the scope of this paper.

Increased mortality rates after elective surgery performed on a Friday have also been reported in other studies. ${ }^{6-8}$ A possible explanation given is inferior quality of care during weekends. Patients who undergo surgery on a Friday may have the disadvantage that their postoperative care occurs during the weekend, which might, 
for example, put them at risk for delayed recognition of complications. In addition, the shift from weekday care toward the suggested inferior weekend care can begin on Friday, possibly influencing surgeries performed on a Friday.

However, these studies showing worse outcomes after Friday surgeries may be limited because of their use of administrative data, which are known to be susceptible to insufficient adjustment for case mix. ${ }^{9}$ As a result, worse outcomes in patients undergoing elective surgery performed on a Friday could be due to their being more severely ill, which was not sufficiently adjusted for in the administrative data. In addition, previous studies investigating patient outcomes in CRC have considered colon and rectal cancer together, whereas patient characteristics, complication risks, and clinical outcomes are different in colon versus rectal cancer. ${ }^{10}$ Furthermore, to provide clues for possible quality improvement, other outcomes require investigation, such as complication risk and FTR, ${ }^{11}$ which have not been addressed previously. The Dutch ColoRectal Audit (DCRA), previously known as the Dutch Surgical Colorectal Audit, is a nationwide clinical audit in the Netherlands with the objective to measure and improve the quality of CRC surgery. It registers detailed clinical data of all CRC procedures, and thus may be better able to adjust for case-mix and make allow valid comparisons of outcomes between patient groups undergoing surgery across different weekdays. ${ }^{12}$ Therefore, the goal of our study was to investigate whether the risk of adverse events after elective colon and rectal cancer surgery differs among weekdays (Monday through Friday) using data from the DCRA.

\section{Patients and Methods}

\section{Study Design and Setting}

Data were prospectively collected from the DCRA, which is a disease-specific audit initiated in 2009 that contains a wide range of variables with specific information regarding patient and tumor characteristics, diagnostics, treatment, complications, and mortality. All Dutch hospitals performing CRC surgery $(\mathrm{N}=88)$ participate in the DCRA. A more detailed description of the DCRA, including a study investigating the effect of weekend surgery among patients undergoing emergency colorectal procedures, is published elsewhere. ${ }^{12-14}$

\section{Study Population}

All patients $(\mathrm{N}=37,402)$ registered in the DCRA for elective surgical resection of primary CRC from 2012 until 2015 were included. Patients with synchronous tumors, treated with the "wait-and-see" approach, undergoing procedures performed solely through transanal endoscopic microsurgery, or without information on date of elective surgery, mortality, or location of the primary tumor were excluded $(n=634)$. Additionally, patients undergoing elective surgery during weekends $(n=152)$ were excluded because elective weekend surgery is not common practice in the Netherlands, and therefore this group may represent a specific selection of patients. The study protocol was presented to the Medical Ethics Committee of the Leiden University Medical Center, which declared that no ethical approval was necessary for this type of study under Dutch law and waived the need for informed written consent from patients (P16.243).

\section{Data and Definitions}

Outcome measures were mortality, severe complications, and FTR. Mortality was defined as postoperative death in the hospital during the same admission or within 30 days after resection. Severe complication was defined as any postoperative complication leading to a prolonged hospital stay of $>14$ days, a reintervention, or mortality. Patients with a severe complication who died during the same admission or within 30 days after resection were defined as FTR. These definitions have also been used in previous DCRA studies.,11,13-15

Case-mix variables used to adjust outcomes for both colon and rectal cancer were age, sex, body mass index, Charlson comorbidity score, American Society of Anesthesiologists classification score, presence of metastasis, preoperative complications, and additional resection for metastasis or locally advanced tumor. Specific to colon cancer, the pathologic tumor stage and location of the primary tumor were added to the case-mix variables, and specific to rectal cancer, the clinical tumor stage, type of surgery (low anterior resection or abdominal perineal resection), and neoadjuvant therapy were added. These case-mix variables have been previously shown to affect patient outcomes and hospital comparisons and are thus part of the standard DCRA case-mix models. ${ }^{13}$

\section{Statistical Analysis}

First, chi-square tests were used to compare patient characteristics by day of elective surgery (Monday through Friday), separately for colon and rectal cancer, given the known differences in complication risks and patient characteristics. ${ }^{10}$ Crude rates were calculated for the 3 outcomes-mortality, severe complications, and FTR - by day of surgery to investigate whether there was a trend across weekdays, as found in previous research. Subsequently, logistic regression was used to examine the unadjusted and adjusted odds ratios and $95 \%$ confidence intervals by day of surgery using Monday as the reference category, as in previous studies.,5,16 Analyses were adjusted for the case-mix variables mentioned earlier. Goodness of fit of the adjusted models was assessed using C-statistics by calculating the area under the 


\section{Table 1. Characteristics of Patients With Colon Cancer}

\begin{tabular}{|c|c|c|c|c|c|c|}
\hline & & & Day of Surgery & & & \\
\hline & Monday (\%) & Tuesday (\%) & Wednesday (\%) & Thursday (\%) & Friday (\%) & $P$ Value ${ }^{a}$ \\
\hline Total elective procedures, $\mathrm{N}$ & 4,820 & 5,174 & 5,431 & 4,905 & 4,762 & \\
\hline Male & 53.9 & 54.6 & 52.6 & 52.7 & 54.4 & \\
\hline Female & 46.1 & 45.4 & 47.4 & 47.3 & 45.6 & \\
\hline$\leq 60$ & 14.7 & 14.0 & 14.6 & 14.1 & 14.8 & \\
\hline $61-70$ & 35.6 & 35.3 & 34.1 & 34.4 & 33.5 & \\
\hline $71-80$ & 33.9 & 33.6 & 35.1 & 34.3 & 35.3 & \\
\hline$\geq 81$ & 15.9 & 17.1 & 16.2 & 17.2 & 16.4 & \\
\hline $\mathrm{BMI}, \mathrm{kg} / \mathrm{m}^{2}$ & & & & & & .009 \\
\hline$\geq 30$ & 18.6 & 18.0 & 18.9 & 18.0 & 19.6 & \\
\hline Charlson comorbidity score & & & & & & NS \\
\hline 0 & 48.9 & 49.4 & 47.8 & 47.7 & 47.7 & \\
\hline 1 & 23.2 & 23.2 & 23.8 & 23.0 & 23.5 & \\
\hline$\geq 2$ & 28.0 & 27.4 & 28.4 & 29.2 & 28.8 & \\
\hline ASA score & & & & & & NS \\
\hline$|-| \mid$ & 75.9 & 77.0 & 76.9 & 76.5 & 77.4 & \\
\hline III & 22.8 & 22.1 & 22.3 & 22.6 & 21.4 & \\
\hline IV-V & 1.3 & 0.9 & 0.8 & 1.0 & 1.2 & \\
\hline Metastasis & & & & & & .009 \\
\hline No & 91.3 & 90.9 & 89.4 & 90.6 & 90.3 & \\
\hline Yes & 8.7 & 9.1 & 10.6 & 9.4 & 9.7 & \\
\hline Preoperative tumor complications & & & & & & .011 \\
\hline No & 69.8 & 67.9 & 67.2 & 69.8 & 68.6 & \\
\hline Yes & 30.2 & 32.1 & 32.8 & 30.2 & 31.4 & \\
\hline Additional resection for metastasis & & & & & & .000 \\
\hline No & 96.4 & 96.5 & 95.2 & 96.5 & 97.1 & \\
\hline Yes & 3.6 & 3.5 & 4.8 & 3.5 & 2.9 & \\
\hline Additional resection for locally advanced tumor & & & & & & NS \\
\hline No & 91.9 & 90.7 & 91.1 & 91.6 & 90.9 & \\
\hline Yes & 8.1 & 9.3 & 8.9 & 8.4 & 9.1 & \\
\hline
\end{tabular}

Abbreviations: ASA, American Society of Anesthesiologists; BMI, body mass index; NS, not significant.

bPathologic tumor stage. 
Table 1. Characteristics of Patients With Colon Cancer (cont.)

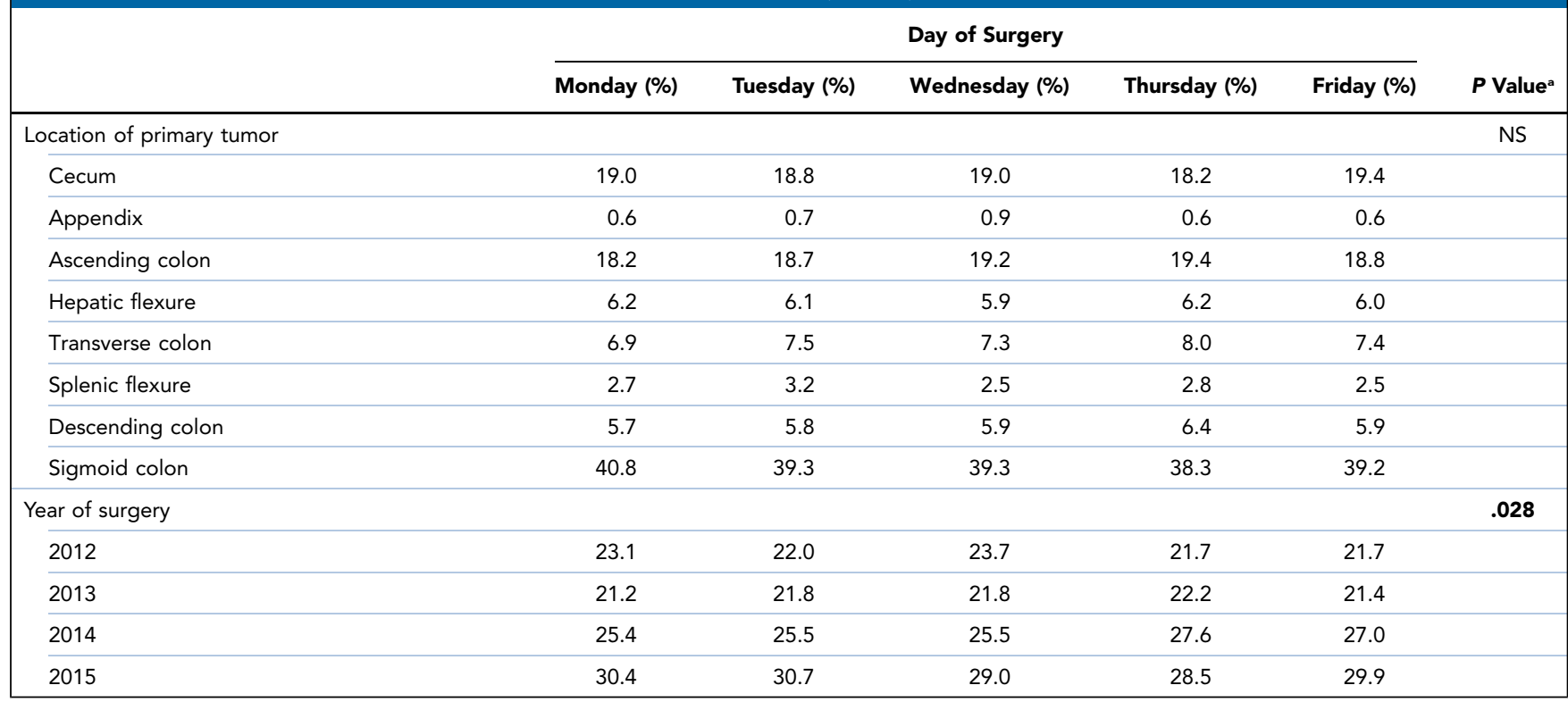

Abbreviations: ASA, American Society of Anesthesiologists; BMI, body mass index; NS, not significant.

abold indicates statistical significance.

bPathologic tumor stage.

receiver operating characteristic curve. Sensitivity analysis was performed to compare Friday surgery versus Monday-through-Thursday surgery to examine differences between surgeries performed on a Friday versus any other weekday. Results were considered statistically significant at $P<.05$. Statistical analyses were performed using SPSS version 23 (IBM Corporation).

\section{Results}

\section{Patient Characteristics}

A total of 36,616 patients were included in the analyses, with 25,092 (68.5\%) undergoing elective colon cancer surgery and 11,524 (31.5\%) undergoing elective rectal cancer surgery. Among those who had elective colon cancer surgery, significant differences in patient characteristics were found by day of surgery for body mass index, metastasis, preoperative tumor complications, additional resections for metastasis, and year of surgery (Table 1). Among patients who underwent elective rectal cancer surgery, significant differences in patient characteristics were found by day of surgery for tumor stage, preoperative tumor complications, additional resection for metastasis and locally advanced tumor, neoadjuvant therapy, surgical procedure, and year of surgery (Table 2).

\section{Elective Surgery}

For colon cancer, elective surgeries performed on Thursday had the highest mortality rate (2.3\%), Tuesday surgeries had the highest severe complication rate (15.5\%), and Friday surgeries had the highest FTR rate (15.1\%), without a clear increasing trend observed across the weekdays (Table 3). After adjustment for case mix, no significant increased risks were seen for any of the outcomes by day of elective surgery compared with Monday surgery.

Among patients with rectal cancer, elective surgeries performed on Wednesday had the highest mortality rate (1.5\%), surgeries performed on Monday and Wednesday had the highest severe complication rates (both 21.8\%), and Tuesday surgeries had the highest FTR rate $(6.8 \%)$ (Table 3). Again, no clear trend was observed across weekdays. However, after adjustment for case mix, severe complication risk was significantly lower for elective Friday surgeries than for Monday surgeries, but the C-statistic indicated that the model did not fit very well.

Similar results were found when comparing Friday surgery with surgery performed on Monday through Thursday, with a lower severe complication rate for rectal cancer surgery performed on a Friday. Slight differences in the odds ratios were observed, but the direction of the association and the model fit remained the same (data not shown).

\section{Discussion}

Given findings of previous studies showing a significantly higher mortality risk after elective surgery performed on a Friday, many wonder whether care delivered to patients should be reorganized to ensure good outcomes across the entire week. We used prospectively collected data from the DCRA to examine the risk of mortality, severe complications, and FTR by day of surgery (Monday 


\section{Table 2. Characteristics of Patients With Rectal Cancer}

\begin{tabular}{|c|c|c|c|c|c|c|}
\hline & \multicolumn{5}{|c|}{ Day of Surgery } & \multirow[b]{2}{*}{$P$ Value ${ }^{a}$} \\
\hline & Monday (\%) & Tuesday (\%) & Wednesday (\%) & Thursday (\%) & Friday (\%) & \\
\hline Total elective procedures, $\mathrm{N}$ & 2,458 & 2,537 & 2,462 & 2,295 & 1,772 & \\
\hline Male & 64.1 & 64.7 & 63.3 & 63.9 & 60.8 & \\
\hline Female & 35.9 & 35.3 & 36.7 & 36.1 & 39.2 & \\
\hline$\leq 60$ & 22.8 & 23.0 & 24.0 & 22.0 & 23.1 & \\
\hline $61-70$ & 37.6 & 38.2 & 37.0 & 36.6 & 37.5 & \\
\hline $71-80$ & 29.6 & 29.3 & 29.1 & 30.7 & 28.8 & \\
\hline$\geq 81$ & 9.9 & 9.5 & 9.9 & 10.8 & 10.5 & \\
\hline $\mathrm{BMI}, \mathrm{kg} / \mathrm{m}^{2}$ & & & & & & NS \\
\hline$\geq 30$ & 16.5 & 16.3 & 17.7 & 16.1 & 16.5 & \\
\hline Charlson comorbidity score & & & & & & NS \\
\hline 0 & 56.2 & 56.6 & 55.5 & 57.3 & 56.4 & \\
\hline 1 & 21.0 & 21.6 & 20.1 & 19.9 & 20.8 & \\
\hline$\geq 2$ & 22.7 & 21.8 & 24.4 & 22.8 & 22.9 & \\
\hline ASA score & & & & & & NS \\
\hline$|-| \mid$ & 83.9 & 84.9 & 83.0 & 82.2 & 84.0 & \\
\hline III & 15.5 & 14.6 & 16.4 & 17.2 & 15.6 & \\
\hline IV-V & 0.6 & 0.5 & 0.7 & 0.6 & 0.4 & \\
\hline Metastasis & & & & & & NS \\
\hline No & 93.6 & 93.3 & 93.1 & 93.6 & 93.5 & \\
\hline Yes & 6.4 & 6.7 & 6.9 & 6.4 & 6.5 & \\
\hline Preoperative tumor complications & & & & & & .005 \\
\hline No & 76.9 & 75.8 & 73.8 & 78.2 & 75.1 & \\
\hline Yes & 23.1 & 24.2 & 26.2 & 21.8 & 24.9 & \\
\hline Additional resection for metastasis & & & & & & .011 \\
\hline No & 96.5 & 97.1 & 96.1 & 97.3 & 97.8 & \\
\hline Yes & 3.5 & 2.9 & 3.9 & 2.7 & 2.2 & \\
\hline Additional resection for locally advanced tumor & & & & & & .001 \\
\hline No & 91.8 & 92.4 & 92.1 & 92.5 & 95.1 & \\
\hline Yes & 8.2 & 7.6 & 7.9 & 7.5 & 4.9 & \\
\hline
\end{tabular}

aBold indicates statistical significance.

${ }^{\mathrm{b} C l i n i c a l}$ tumor stage. 
Table 2. Characteristics of Patients With Rectal Cancer (cont.)

\begin{tabular}{|c|c|c|c|c|c|c|}
\hline & & & Day of Surgery & & & \\
\hline Neoadjuvant therapy & & & & & & .004 \\
\hline No & 27.1 & 27.6 & 28.5 & 29.9 & 28.3 & \\
\hline Short-course radiotherapy & 33.4 & 36.4 & 33.3 & 35.5 & 37.0 & \\
\hline Chemoradiotherapy & 39.5 & 36.0 & 38.2 & 34.6 & 34.8 & \\
\hline LAR & 1.6 & 1.0 & 1.3 & 1.2 & 1.2 & \\
\hline APR & 67.8 & 71.5 & 68.1 & 71.4 & 71.2 & \\
\hline Other & 30.6 & 27.4 & 30.6 & 27.5 & 27.7 & \\
\hline Year of surgery & & & & & & .000 \\
\hline 2012 & 21.8 & 23.5 & 25.4 & 22.8 & 24.9 & \\
\hline
\end{tabular}

Abbreviations: APR, abdominal perineal resection; ASA, American Society of Anesthesiologists; BMI, body mass index; LAR, low anterior resection; NS, not significant.

aBold indicates statistical significance.

bClinical tumor stage.

through Friday) for elective colon and rectal cancer surgery. No elevated mortality risk was found, but instead the results showed lower severe complication risk after Friday elective surgery for rectal cancer and no significant differences for colon cancer.

In contrast with previous studies, ${ }^{4-8}$ our study showed no significantly increased risk of mortality when elective surgery was performed on Friday. Interestingly, the Global Comparator study by Ruiz et $\mathrm{al}^{6}$ found a "Friday effect," especially for the 6 Dutch hospitals participating in the study. These findings might be explained by the fact that they used administrative data and that the participating Dutch academic hospitals are not representative of all Dutch hospitals. Our study, however, used clinical DCRA data and included all Dutch hospitals. Dubois et $\mathrm{al}^{17}$ also did not find differences for elective surgeries performed on Friday compared with Monday; however, they excluded patients undergoing surgery outside office hours (also when it was a weekday). In our study, these patients might have been included because time of surgery is not recorded in DCRA data, but we still did not find any increased risk for Friday surgery.

Remarkably, we found significantly lower risk for severe complications when elective rectal cancer surgery was performed on a Friday, but this must be interpreted cautiously, given the fit of the model. However, if this were true, one possible reason for these findings might be that, on a Friday, less experienced surgeons are operating on patients with less technically complex disease. This explanation seems supported by 2 previous studies showing that working experience among surgeons is lowest on Fridays, ${ }^{16,17}$ with junior and midlevel surgeons performing more surgeries on Fridays and senior surgeons performing fewer. ${ }^{16}$ When it is common to have less experienced surgeons working on a Friday, it is also likely that patients with less technically complex disease are scheduled for surgery on a Friday, and these patients also may have a lower risk for adverse events. Consistent with this explanation, our study showed slightly lower tumor stage and fewer additional resections for metastasis and locally advanced tumor among patients undergoing rectal cancer surgery on a Friday compared with other weekdays. This could reflect how the lower risk for severe complications after elective surgery performed on a Friday is due to selection of less complex patients. Another possible reason for our findings may be differences in discharge policies. Whether the findings are caused by, on average, less experienced surgeons working on a Friday, less complex disease, or other factors, such as organization of care, should be further explored.

A limitiation of this study may be that information on time of surgery was not available, and therefore it is possible that the study population included patients who received surgery outside office hours (ie, during evenings and nights). Because elective surgery performed outside office hours is not common, similar to elective weekend surgeries, this might indicate a specific selection of patients. Use of the DCRA in our study could be considered a strength, particularly because of the available clinical variables for better case-mix adjustment in contrast to 
Table 3. Outcomes of Patients With Colon and Rectal Cancer

\begin{tabular}{|c|c|c|c|c|c|c|}
\hline & \multicolumn{5}{|c|}{ Day of Surgery } & C-Statistic \\
\hline \multicolumn{7}{|l|}{ Colon } \\
\hline Elective procedures, $n$ & 4,820 & 5,174 & 5,431 & 4,905 & 4,762 & \\
\hline 30-d mortality, n (\%) & $94(2.0)$ & $105(2.0)$ & $96(1.8)$ & $111(2.3)$ & $104(2.2)$ & \\
\hline Unadjusted OR $(95 \% \mathrm{Cl})$ & Ref & $1.04(0.79-1.38)$ & $0.91(0.68-1.21)$ & $1.16(0.88-1.54)$ & $1.12(0.85-1.49)$ & \\
\hline Severe complications, $\mathrm{n}(\%)$ & $726(15.1)$ & $804(15.5)$ & $761(14.0)$ & $742(15.1)$ & $688(14.4)$ & \\
\hline Unadjusted OR $(95 \% \mathrm{Cl})$ & Ref & $1.04(0.93-1.16)$ & $0.92(0.82-1.03)$ & $1.01(0.90-1.12)$ & $0.95(0.85-1.07)$ & \\
\hline Adjusted OR $(95 \% \mathrm{Cl})$ & Ref & $1.03(0.92-1.15)$ & $0.92(0.82-1.03)$ & $0.99(0.89-1.11)$ & $0.95(0.85-1.06)$ & .623 \\
\hline Failure to rescue, ${ }^{a} \mathrm{n}(\%)$ & $94(12.9)$ & $105(13.1)$ & $96(12.6)$ & $111(15.0)$ & $104(15.1)$ & \\
\hline Unadjusted OR $(95 \% \mathrm{Cl})$ & Ref & $1.01(0.75-1.36)$ & $0.97(0.72-1.32)$ & $1.18(0.88-1.59)$ & $1.20(0.89-1.62)$ & \\
\hline 30-d mortality, n (\%) & $34(1.4)$ & $36(1.4)$ & $36(1.5)$ & $28(1.2)$ & $22(1.2)$ & \\
\hline Unadjusted OR (95\% Cl) & Ref & $1.03(0.64-1.65)$ & $1.06(0.66-1.70)$ & $0.88(0.53-1.46)$ & $0.90(0.52-1.54)$ & \\
\hline Adjusted OR (95\% Cl) & Ref & $1.10(0.68-1.80)$ & $1.05(0.64-1.72)$ & $0.93(0.56-1.57)$ & $0.94(0.53-1.64)$ & .791 \\
\hline Severe complications, n (\%) & $536(21.8)$ & $527(20.8)$ & $537(21.8)$ & 489 (21.3) & $338(19.1)$ & \\
\hline Unadjusted OR $(95 \% \mathrm{Cl})$ & Ref & $0.94(0.82-1.08)$ & $1.00(0.87-1.15)$ & $0.97(0.85-1.12)$ & $0.85(0.73-0.98)$ & \\
\hline Adjusted OR $(95 \% \mathrm{Cl})$ & Ref & $0.92(0.80-1.06)$ & $0.97(0.85-1.11)$ & $0.95(0.83-1.09)$ & $0.84(0.72-0.97)$ & .565 \\
\hline Failure to rescue, ${ }^{a} \mathrm{n}(\%)$ & $34(6.3)$ & $36(6.8)$ & $36(6.7)$ & $28(5.7)$ & $22(6.5)$ & \\
\hline Unadjusted OR (95\% Cl) & Ref & $1.08(0.67-1.76)$ & $1.06(0.65-1.72)$ & $0.90(0.54-1.50)$ & $1.03(0.59-1.79)$ & \\
\hline Adjusted OR $(95 \% \mathrm{Cl})$ & Ref & $1.27(0.76-2.12)$ & $1.18(0.71-1.98)$ & $1.02(0.59-1.76)$ & $1.17(0.65-2.10)$ & .760 \\
\hline
\end{tabular}

Abbreviation: OR, odds ratio.

aRates and ORs are based on the rates of severe complications.

previous studies that mostly used administrative data. However, it is important to note that our findings did not change after adjusting for case mix, thereby suggesting that these additional clinical data may not be that important for case-mix adjustments compared with administrative data on CRC surgery. Lastly, we assessed colon and rectal cancer surgery separately, given that patient characteristics, complication risks, and clinical outcomes are different. ${ }^{10}$ By separating CRC surgery in our analysis, we believe that our results give a more adequate picture of how the day of elective surgery is associated with differences in patient outcomes.

\section{Conclusions}

In contrast to previous studies, our study found that mortality risk associated with elective surgery did not differ across weekdays for colon and rectal cancer surgery in the Netherlands. We observed lower severe complication risk after elective rectal cancer surgery performed on Fridays compared with Mondays, perhaps because of a selection of patients with less complex disease scheduled for surgery on a Friday, but explanatory factors for these findings should be further investigated.

\section{Acknowledgments}

The authors wish to thank all surgeons, registrars, physician assistants, and administrative nurses who registered the patients in the DCRA, as well as the DRCA group.

Submitted October 22, 2018; accepted for publication February 6, 2019

Author contributions: Study design: Guicherit, Marang-van de Mheen. Data analysis and interpretation: Huijts, Dekker, van Groningen, Marang-van de Mheen. Manuscript preparation: Huijts, Marang-van de Mheen. Critical revision: Guicherit, Dekker, van Groningen, van Bodegom-Vos, Bastiaannet, Govaert, Wouters, Marang-van de Mheen.

Disclosures: The authors have disclosed that they have no financial interests, arrangements, or affiliations with the manufacturers of any products discussed in this article or their competitors.

Funding: This study was funded by an unrestricted grant from the Dutch Cancer Society (UL2015-7772). The Dutch Cancer Society did not influence the article in any way.

Correspondence: Perla J. Marang-van de Mheen, PhD, Department of Biomedical Data Sciences, Medical Decision Making, Leiden University Medical Center, Albinusdreef 2, 2333 ZA, Leiden, the Netherlands. Email: p.j.marang@lumc.nl 


\section{References}

1. Paun BC, Cassie S, MacLean AR, et al. Postoperative complications following surgery for rectal cancer. Ann Surg 2010;251:807-818.

2. Henneman D, Ten Berge MG, Snijders HS, et al. Safety of elective colorectal cancer surgery: non-surgical complications and colectomies are targets for quality improvement. J Surg Oncol 2014;109:567-573.

3. Govaert JA, Fiocco M, van Dijk WA et al. Costs of complications after colorectal cancer surgery in the Netherlands: building the business case for hospitals. Eur J Surg Oncol 2015;41:1059-1067.

4. Smith SA, Yamamoto JM, Roberts DJ, et al. Weekend surgical care and postoperative mortality: a systematic review and meta-analysis of cohort studies. Med Care 2018;56:121-129.

5. Aylin P, Alexandrescu R, Jen $\mathrm{MH}$, et al. Day of week of procedure and 30 day mortality for elective surgery: retrospective analysis of hospital episode statistics. BMJ 2013;346:f2424

6. Ruiz M, Bottle A, Aylin PP. The Global Comparators project: international comparison of 30-day in-hospital mortality by day of the week. BMJ Qual Saf 2015;24:492-504.

7. Vohra RS, Pinkney T, Evison F, et al. Influence of day of surgery on mortality following elective colorectal resections. Br J Surg 2015;102:1272-1277.

8. Zare MM, Itani KM, Schifftner TL, et al. Mortality after nonemergent major surgery performed on Friday versus Monday through Wednesday. Ann Surg 2007;246:866-874

9. Lilford RJ, Chen YF. The ubiquitous weekend effect: moving past proving it exists to clarifying what causes it. BMJ Qual Saf 2015;24:480-482.
10. van der Sijp MP, Bastiaannet E, Mesker WE, et al. Differences between colon and rectal cancer in complications, short-term survival and recurrences. Int J Colorectal Dis 2016;31:1683-1691.

11. Henneman D, van Leersum NJ, Ten Berge M, et al. Failure-to-rescue after colorectal cancer surgery and the association with three structural hospital factors. Ann Surg Oncol 2013;20:3370-3376.

12. Van Leersum NJ, Snijders HS, Henneman D, et al. The Dutch Surgical Colorectal Audit. Eur J Surg Oncol 2013;39:1063-1070.

13. Kolfschoten NE, Marang van de Mheen PJ, Gooiker GA, et al. Variation in case-mix between hospitals treating colorectal cancer patients in the Netherlands. Eur J Surg Oncol 2011;37:956-963.

14. Huijts DD, van Groningen JT, Guicherit OR, et al. Weekend effect in emergency colon and rectal cancer surgery: a prospective study using data from the Dutch ColoRectal Audit. J Natl Compr Canc Netw 2018;16: 735-741.

15. Henneman D, Snijders HS, Fiocco M, et al. Hospital variation in failure to rescue after colorectal cancer surgery: results of the Dutch Surgical Colorectal Audit. Ann Surg Oncol 2013;20:2117-2123.

16. Ruiz M, Bottle A, Aylin PP. Exploring the impact of consultants' experience on hospital mortality by day of the week: a retrospective analysis of hospital episode statistics. BMJ Qual Saf 2016;25:337-344.

17. Dubois L, Vogt K, Vinden C, et al. Association between day of the week of elective surgery and postoperative mortality. CMAJ 2017 189:E303-309.

\title{
Now Accepting Call for Abstracts
}

\section{March $20-22$
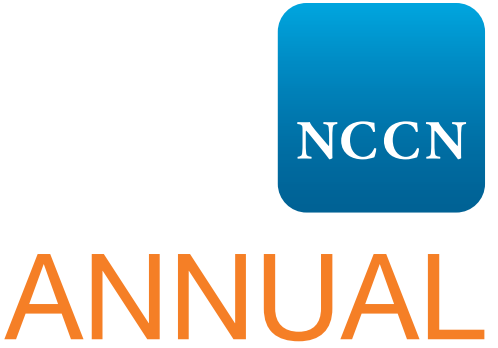 \\ CONF
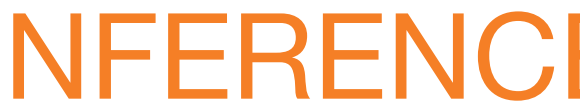

- Celebrating 25 years of NCCN

\author{
Rosen Shingle Creek • Orlando, Florida
}

NCCN.org/conference 